\title{
Orientación estratégica y desempeño en un proceso de desarrollo de productos Alfarería en Santa María Atzompa, Oaxaca, México
}

\author{
María Luisa Domínguez Hernández* \\ José de la Paz Hernández Girón* \\ Dora Lilia Guzmán Cruz*
}

\begin{abstract}
Resumen
Este trabajo tiene como objetivo explicar cómo en un proceso de desarrollo de producto la orientación estratégica afecta el desempeño de 19 negocios de alfarería de Santa María Atzompa, Oaxaca. El proceso de desarrollo de productos duró quince meses, en el que se utilizaron procedimientos muy detallados para facilitar la integración de las bases del conocimiento adquirido mediante la capacitación. En este documento se pone a prueba un modelo de investigación donde se determina que el desempeño de los negocios de artesanía, medido por el monto de las ventas y de las utilidades, se ve afectado por el impacto de las variables antecedentes al desempeño. La orientación a la calidad y hacia la innovación son las variables con mayor efecto sobre el desempeño, mientras que la orientación al mercado y a los consumidores sólo afectan las utilidades de los negocios.
\end{abstract}

Palabras clave: orientación estratégica, desempeño, artesanía, capacitación.

Fecha de recepción: 02/10/2007

Fecha de aceptación: 30/01/2008

The Process of Product Development, its Strategic Orientation and Performance in Santa Maria Atzompa's handcraft business

\begin{abstract}
The aim of this paper is to show how during the process of product development a strategic orientation had an impact on the performance of nineteen handcraft firms located in Santa Maria Atzompa. The product development process lasted fifteen months and it consisted of following detailed procedures that facilitated the integration of acquired knowledge bases. This knowledge was gained through training activities. The paper tested a model that showed how the performance of the handcraft firms was affected by variables prior to performance. Performance was measured by means of sales and profits levels. The evidence found that quality
\end{abstract}

*Profesores-investigadores del Instituto Politécnico Nacional.

Correos electrónicos: mdominguezh3@hotmail.com, jgiron4@hotmail.com, doligu0531@hotmail.com

No. 225, mayo-agosto 2008: 79-101 
María Luisa Domínguez Hernández, José de la Paz Hernández Girón y Dora Lilia Guzmán Cruz

and innovation orientation variables had the highest effect on performance while market and customer orientation only impacted on profits.

Key words: strategic orientation, performance, handcraft, training.

\section{Introducción}

T a orientación estratégica es considerada una parte importante de la cultura orga_nizacional (Hunt y Morgan, 1995); ésta define las distintas culturas organizacionales que ponen al cliente en el centro del pensamiento estratégico y operacional de la empresa. De igual manera, tiene un impacto en los vendedores mediante la satisfacción en el trabajo y la orientación al cliente (Mavondo, 1999).

Aragón y Sánchez $(2005,290)$ suponen que las empresas con orientaciones defensivas, prospectivas o analizadoras pueden conducir a un desempeño satisfactorio, no así las reactivas debido a la falta de consistencia interna. Para medir el desempeño usaron dos medidas diferentes: Retorno sobre la inversión (ROI) y la percepción de la situación de la empresa con respecto a sus competidores. Las empresas pequeñas que siguen estrategias prospectivas son las que mejor toman ventaja de los recursos internos de la empresa. Las orientaciones estratégicas provocan que el desempeño de la empresa sea diferente y las empresas con orientación prospectiva superen al resto debido a su mayor proactividad y capacidad para adaptarse. Resaltan en sus conclusiones que las empresas pequeñas que siguen estrategias prospectivas tienen un comportamiento estratégico y de dirección más similar al de empresas grandes.

Las estrategias de mercadotecnia como factores de éxito son una perspectiva que conduce el estilo de vida de los artesanos, experimentando, aprendiendo, desaprendiendo, con comprobación continua y reaccionando al ambiente. La estrategia en el sector artesanal es una interpretación del mundo que puede modificarse en función de los planes y programas de producción y mercadotecnia para asegurar el éxito de los productores de artesanía (Hernández et al., 2007, 23). Pero hace falta explicar cómo, en un proceso de desarrollo de producto, la orientación estratégica afecta el desempeño de los artesanos (ver figura 1). 
Orientación estratégica y desempeño en un proceso de desarrollo de productos

Alfarería en Santa María Atzompa, Oaxaca, México

Figura 1

Modelo de investigación propuesto

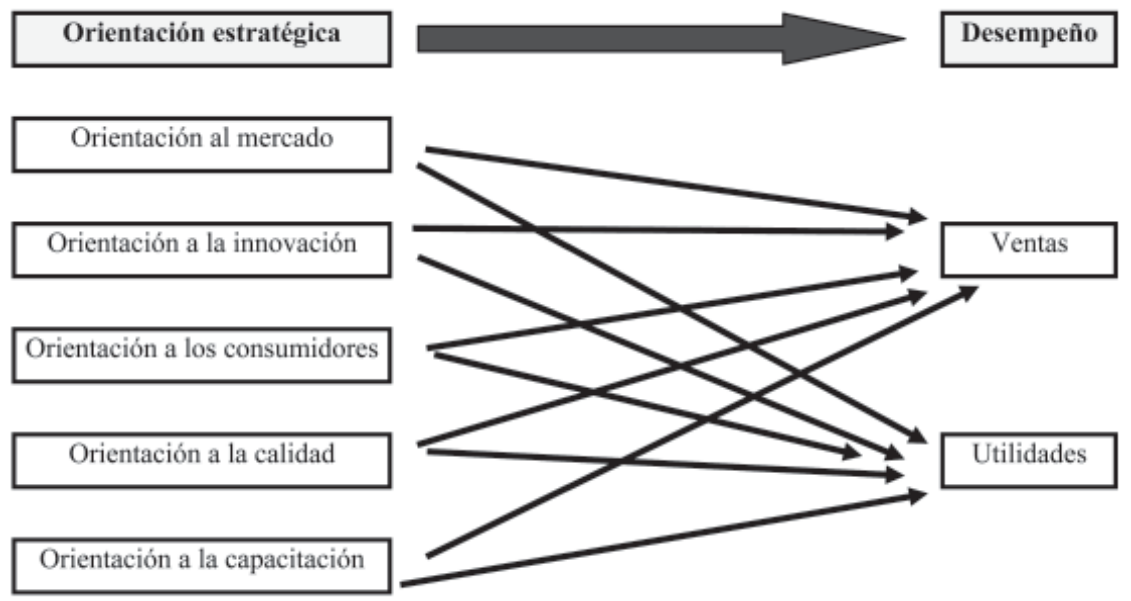

\section{Orientación estratégica}

La evolución del enfoque de estrategias, dentro de lo que se considera administración estratégica, empieza desde las escuelas de negocios hasta las teorías de recursos y capacidades, continuando con las estrategias competitivas y finalizando en la complejidad del mundo actual donde se aborda el concepto de estrategia con estudios de factores y variables flexibles y dinámicas (Mintzberg, 1987; Barney, 1991; Morgan y Strong, 1998; Morales y Pech, 2000; Aragón y Sánchez, 2005; Linares y Acevedo, 2006; Brouthers et al., 2007 y Ho y Huang, 2007). Existen diferentes enfoques de cómo se han estudiado las estrategias, iniciando con las estrategias genéricas de Ansoff en las que incluye la penetración de mercados; desarrollo de producto; desarrollo de mercados y diversificación. Siguiendo con Porter — cuyo enfoque ha encontrado una amplia aceptación en la administración estratégica orientada a la forma cómo una empresa plantea sus actividades y a su manera de competir en un sector industrial- utiliza estrategias que también llama genéricas: liderazgo en costos, diferenciación y de enfoque. Otros enfoques han centrando su visión en las capacidades de la empresa y la manera en que los recursos permiten aplicar y crear una ventaja competitiva (Barney, 1991). Para Linares y Acevedo (2006: 51), las estrategias pueden ser divididas en corporativas, globales, de negocio, funcionales y tecnológicas. Otra tipología de las estrategias se centra en los conceptos de estrategias funcionales, de las que existen tantas como funciones o áreas de negocios existen en una empresa. 
Según Morgan y Strong (1998, 1053-1055), el término de orientación estratégica ha sido utilizado como estrategia competitiva, predisposición estratégica, ajuste estratégico, empuje estratégico y alternativa estratégica. La orientación estratégica puede definirse "cómo la organización usa la estrategia para adaptar y/o cambiar aspectos de su entorno para lograr un ajuste más favorable". Estos autores clasifican la orientación estratégica en dimensiones como agresiva, analizadora, defensiva, futurista, proactiva y arriesgada.

Para Aragón y Sánchez $(2005,290)$, la tipología sobre orientación estratégica de Miles y Snow, sugerida en 1978, ha tenido uno de los efectos más difundidos porque se considera única y considera a la organización como un sistema completo e integrado en interacción dinámica con sus ambientes. Esta tipología está constituida por tres premisas: 1) Las empresas exitosas desarrollan un método sistemático de alineación con su ambiente, mientras responden al ciclo adaptable. 2) Pueden identificarse cuatro orientaciones estratégicas en cada industria: defensiva, prospectiva, analizadora y reactiva. 3) Las estrategias defensiva, prospectiva y analizadora pueden conducir a un desempeño satisfactorio; mientras que la reactiva no puede debido a su falta de consistencia interna.

También Brouthers y et al. $(2007,228)$ conceptualizan la orientación estratégica como una variable continua cuyos extremos son, por un lado, las estrategias proactivas o muy agresivas y, por el otro, las estrategias defensivas o menos agresivas.

Este enfoque de orientación estratégica se centra en la agresividad, que es un concepto multifacético determinado por los niveles de análisis, defensa, futuro, riesgo y proactividad de la estrategia, sin especificar de cuál estrategia se trata. Por su parte, Morales y Pech $(2000,51)$ argumentan que la estrategia de la empresa, en administración, tiene como objetivo la creación de ventaja competitiva y concluyen que "La estrategia derivará en lineamientos o políticas de asignación de recursos con base en los objetivos y metas de la empresa, apoyándose en sus puntos fuertes y en función de las características del medio ambiente". Se refieren a cinco políticas funcionales: producción, mercadotecnia, investigación y desarrollo, finanzas y recursos humanos.

Kohli y Jaworski $(1990,4)$ consideran que la orientación al mercado tiene un enfoque al consumidor y que es su elemento central. El punto de vista más tradicional implica obtener información de los consumidores acerca de sus necesidades y preferencias. Además, refieren a la orientación al mercado como la encargada en la organización de la generación, diseminación y responsable de la inteligencia de mercado. Definen la orientación al mercado como la generación, en todo lo ancho de la organización, de la inteligencia de mercado pertinente para las necesidades presentes y futuras de 
Orientación estratégica y desempeño en un proceso de desarrollo de productos

Alfarería en Santa María Atzompa, Oaxaca, México

los consumidores, la diseminación de esa inteligencia a través de los departamentos y la responsabilidad para lograrlo.

Entre las características manejadas en la orientación estratégica por Salavou et al. $(2004,1100)$ manifiestan que la orientación al mercado ha tenido un gran interés en la literatura de administración y mercadotecnia, por lo que manejan como dimensión de la orientación estratégica: la orientación al mercado, la orientación al cliente y la implementación de la estrategia de manejo del mercado. La orientación al mercado se entiende como un juego de comportamientos específicos y actividades que expresan hasta qué punto una empresa entiende y responde a las necesidades del cliente.

También Ho y Huang $(2007,298)$ argumentan que la orientación al mercado se describe como las actividades conductuales y de procesos de todo el negocio, dividida en cinco componentes distintos: orientación a los consumidores, orientación a la fuerza de ventas, orientación a los competidores, orientación al ambiente y orientación interfuncional (interés, comunicación, negociaciones y manejo de problemas departamentales). Y si como dice Morgan y Strong $(1998,1067)$ la orientación al mercado ha sido conceptualizada como la orientación estratégica de las empresas comerciales, entonces sólo haría falta la orientación a la producción para completar la orientación estratégica de la empresa. Además, estos últimos concluyen que deben hacerse esfuerzos para animar a los ejecutivos a pensar en la aplicación de la estrategia más allá de un enfoque per se y reconocer que lo más importante es la forma de la estrategia competitiva, donde la orientación al mercado juega un papel importante en su determinación.

Por lo anterior, es posible argumentar que existen distintos componentes de la orientación estratégica con base en los cuales se establecen las estrategias organizacionales o las estrategias funcionales de la organización, que al final sirven para crear ventaja competitiva: orientación a la calidad, a la innovación, a la producción, al consumidor, al mercado y a la capacitación.

En la orientación a la producción se pone énfasis en la calidad técnica del producto y en la innovación porque no hay consumidor que se resista a un buen producto que tenga un precio razonable. Cuando un producto está bien elaborado, es novedoso y tiene un precio justo, el esfuerzo de mercadotecnia tiende a ser menor, inclusive se piensa que ese esfuerzo es innecesario.

La orientación de mercado tiene una naturaleza genérica. El trabajo sobre la orientación al mercado da énfasis a la necesidad de las empresas para entender claramente a los clientes, competidores y responsables de la cadena de mercado, de tal manera que permita sistemáticamente interpretar y responder a las circunstancias de los mercados 
actuales y probables. Por consiguiente, el papel de la orientación al mercado es determinar la forma de la estrategia competitiva, más que centrarse en su aplicación.

Sin embargo, para el éxito de una empresa, no sólo hay que centrarse en investigar el mercado, hay que asegurarse de que los procesos que la organización lleva adelante internamente se alineen desde el cliente y no únicamente a partir del producto ofrecido.

En la orientación al consumidor la tendencia es que la empresa se enfoque a lograr que el producto o servicio se ajuste bien a las necesidades del consumidor y que también se preocupe por un manejo de precios como respuesta a sus sugerencias, de tal manera que no sea necesario emplear una activa fuerza de ventas, sino que se venda prácticamente solo.

Si la orientación estratégica se enfoca al medioambiente interno y al externo, —como una forma de lograr más fácilmente la supervivencia y que se optimice el logro de los objetivos organizacionales- puede darse el caso que estos objetivos se alcancen más eficazmente, utilizando las fuerzas (recursos humanos) existentes en el interior de la organización, o buscarlas en el exterior. Los problemas actuales como ambientales, económicos, políticos y sociales motivan a las organizaciones para encontrar nuevas formas de administrarse, nuevas formas de competir, de tal modo que se enfoquen en el desarrollo integral de sus recursos humanos.

Aunque en México la capacitación no se ha considerado tradicionalmente como un factor de desarrollo que permita a las empresas dentro de su plan estratégico lograr mejores beneficios y posicionamiento en los mercados, la orientación a la capacitación es una preocupación constante acerca del futuro de la organización; además, es parte de las decisiones al interior de la organización sobre formación, promoción, planes de carrera, reemplazos y retribuciones, decisiones que contribuyen a alcanzar los objetivos logísticos y estratégicos de la organización.

Debido a la dimensión económica podemos decir que está desapareciendo la estabilidad en las estructuras de la organización, sus sistemas, sus procedimientos de trabajo y en sus actividades. La creatividad está a la orden del día, pues existe la necesidad de colocar productos innovadores en el mercado; la orientación estratégica a la capacitación es parte de la planificación proactiva, que conduce a la renovación crecimiento y desarrollo de la fuerza de trabajo (Burack, 1990).

La orientación a la capacitación implica tener un diagnóstico del clima laboral de las diferentes áreas de la empresa, como una consecuencia de su preocupación por los recursos humanos, lo que le proporciona la oportunidad de utilizar las fortalezas o 
Orientación estratégica y desempeño en un proceso de desarrollo de productos

Alfarería en Santa María Atzompa, Oaxaca, México

ventajas comparativas de la empresa, considerando a los empleados como parte integrante del mercado y como un factor fundamental su capacitación.

Según Meza $(2005,78)$, "el maestro artesano convierte el taller artesanal en un espacio de formación en los buenos hábitos de hombría y profesionalidad, y el modelo de capacitación para el trabajo en un promotor de valores y virtudes, con lo que rebasa el mero adiestramiento en el oficio" En el caso del sistema industrial, se concibe a la persona dentro de la empresa como un factor más de la producción, al que habilita únicamente y se la instrumentaliza reduciéndola al hacer. Cuando una organización se enfoca a su fuerza de trabajo, es decir, es plenamente humana, se fomentan actitudes positivas para el desarrollo profesional y personal del trabajador, proporcionándole conocimientos para desempeñar adecuadamente su tarea, y desarrollando en la persona habilidades y destrezas que le permitan hacer bien su trabajo.

Meza $(2005,87)$ al hablar de lo que es la "pedagogía empresarial" considera que ha de promover siempre un modelo de capacitación para el trabajo que tenga como fundamento la idea de formar personas, como principio rector de la educación, contribuyendo al crecimiento de la empresa.

En resumen, la orientación estratégica se define operativamente como el conjunto de estrategias que integran y conducen las acciones necesarias del negocio con el objetivo de promover la creación de un valor superior para todos los involucrados con la empresa. La estrategia ante todo es una serie de acciones que pueden ser preventivas, ofensivas, defensivas o agresivas, resultado de un análisis continuo sin dejar detalle a la improvisación (Mintzberg, 1987). Sus dimensiones serían: orientación a la calidad, a la innovación, al mercado, a los consumidores y a la capacitación del empleado. Todas esas dimensiones coexisten con diferentes niveles de intensidad, conformando una especie de mapa estratégico que actúa como elemento central proporcionando un contexto conceptual accesible a la operación rutinaria de la organización.

La orientación al mercado facilita la claridad de enfoque y visión de la estrategia de la organización, la calidad de la investigación de mercado puede ser sospechosa, o la calidad de la ejecución de los programas de mercadotecnia diseñados en respuesta a la investigación de mercado puede ser pobre (Kohli y Jaworski 1990, 18). Esto indica que la orientación al mercado es parte de la orientación estratégica de la organización e implica que siendo la orientación al mercado una variable, que va de una orientación total al mercado a una orientación pobre, puede dar cabida a que predomine otro tipo de orientación que sea estratégica y que dé lugar a que en la organización haya un mapa estratégico. Ese mapa se puede apreciar en la figura 1 donde los artesanos, sujetos de este trabajo, están más orientados hacia la innovación y menos hacia la calidad, el mercado y la capacitación. 
González $(2003,30)$ también señala que

La Dirección Estratégica ha contribuido notablemente a aclarar que la cuota de mercado no constituye el único rasgo diferencial importante entre las empresas englobadas en el mismo sector. De hecho, en casi cualquier industria es posible observar diferencias en cuanto a la orientación estratégica perseguida por cada una de las empresas que lo integran.

\section{Desempeño}

Existe una multidimensionalidad subyacente a la estructura conceptual de desempeño, la multitud de indicadores utilizada responde a la necesidad de lograr una medida subjetiva fiable (Eddleston et al., 2007). Una medición subjetiva de desempeño en negocios pequeños es común porque hay dificultad para obtener datos y medidas objetivas como las siguientes:

Rentabilidad, medida como: Ganancias por ventas (ROS) = Ingresos netos / Ventas; Ganancias por activos $(\mathrm{ROA})=$ Ingresos netos / Activos totales; Ganancias por inversiones $(\mathrm{ROE})=$ Ingresos netos / Inversión a plazo fijo.

Eficiencia operativa medida como: Eficiencia de ventas $(\mathrm{SALEFF})=$ Ventas / Número de empleados; Eficiencia de ingresos netos $(\mathrm{NIEFF})=$ Ingresos netos / Núm. de empleados.

Inversión de capital medida por: Gasto de capital por ventas $(\mathrm{CESA})=$ Gastos de capital / Ventas; Gasto de capital por activos (CETA) = Gastos de capital / Activos totales. Productividad medida con: Ventas reales $(\mathrm{SAL})=$ Ventas nominales / Índice de precios al consumidor.

Empleo medido por: Empleo total $($ EMPL $)=$ Número total de empleados. $Y$

Solvencia medida como: Deuda vs activos (LEV) = Deuda total / Activos totales; Deuda a largo plazo $v$ s inversión (LEV2) = Deuda a largo plazo / Inversión.

Además, generalmente los negocios pequeños no publican sus datos financieros o no los tienen.

Para evaluar el desempeño organizacional Pleshko y Soulden $(2003,262)$ usan medidas perceptivas sobre las utilidades, evitando el uso de los métodos de contabilidad 
Orientación estratégica y desempeño en un proceso de desarrollo de productos

Alfarería en Santa María Atzompa, Oaxaca, México

que se consideran medidas inconstantes y asociadas con medidas objetivas. Preguntaron sobre el comportamiento de las utilidades en una escala de pobre (1) a excelente (5) con base en cinco aspectos: competidores, metas y expectativas en los años anteriores, potencial de la empresa y crecimiento.

Rodríguez $(2005,41)$ utiliza como medidas de desempeño: los beneficios económicos/rentabilidad (contribución a los beneficios), ventaja competitiva sostenible, un trabajo con suficiente coordinación entre las áreas o departamentos receptores del conocimiento (coordinación organizacional), aprendizaje y/o desarrollo del personal. Concluye que el desempeño de una firma puede ser evaluado desde una perspectiva integral que involucra de una manera amplia los objetivos de la firma.

Marlin et al. $(2007,217)$ utilizan como medidas de desempeño el margen operativo, el retorno sobre activos y las utilidades diarias, utilizan desempeño organizacional como sinónimo de éxito organizacional (229).

Si el éxito de cualquier empresa se centra en lograr ventas y utilidades satisfactorias, y una manera de lograrlo es teniendo una orientación estratégica bien definida, entonces ésta debe estar encaminada a detectar y satisfacer necesidades y deseos como un medio para obtener volúmenes de venta con utilidades.

Una de las formas elementales de medir el desempeño de los negocios de artesanía sería el análisis de las ventas y de las utilidades, que puede hacerse por líneas de productos y por segmentos del mercado, por departamentos, por división, negocio o sector. Para realizar cualquier proceso de análisis es necesaria la comparación y ésta no es posible si no contamos con una referencia contra la cual constatar el valor de un indicador. Esta comparación puede hacerse entre unidades del sector.

\section{Metodología}

Este trabajo es de corte estadístico, se realizaron las observaciones y el análisis de la información utilizando una entrevista estructurada. Primero se contextualiza el proceso de capacitación, se describe la muestra, considerando que los sujetos son todos los que asistieron al proceso y que hay 321 unidades económicas dedicadas a la alfarería; se determinó la estructura conceptual estadística de las variables y con esos datos se presenta una validación estadística de las escalas utilizando un análisis de factores.

Para determinar el mapa estratégico de los artesanos de Santa María Atzompa, se utilizaron los reactivos resultantes de los análisis factoriales; se determinaron las medias de cada variable, pero como tienen diferente número de reactivos las medias no son 
equiparables, entonces con las diferentes medias y sus respectivas desviaciones estándar, se estandarizaron los valores determinando la gran media, que en la gráfica 1 es la constante. Graficando cada media estandarizada de acuerdo con sus respectivos valores se obtuvo el perfil o mapa de las diferentes orientaciones estratégicas.

Dado que la muestra es muy pequeña, la prueba del modelo se realizó con una prueba de medias, utilizando la prueba t de Student.

\section{Contexto del proceso de desarrollo de productos}

La investigación se llevó a cabo dentro de un proceso de desarrollo de productos, que duró quince meses, con un grupo de 19 productores que respondieron a una convocatoria de capacitación, parte de un proceso sistemático de análisis de la capacidad emprendedora de los artesanos en México.

La estructura ocupacional de Santa María Atzompa la integran tres actividades económicas importantes: alfarería, agricultura y comercio. Esta comunidad —que se encuentra ubicada a $10 \mathrm{~km}$. del centro de la ciudad de Oaxaca- se dedica en un $90 \%$ a la alfarería. Las familias de los productores, sujetos de este trabajo, están integradas por un promedio de 5 a 6 personas. La asignación de actividades del proceso de elaboración de piezas de barros se hace de acuerdo con la edad y el sexo, de esta manera los hombres trituran el barro, hacen el lodo y hornean las piezas; mientras las mujeres modelan las piezas, dibujan, graban, pintan y bordan; los niños, por su parte, acarrean las piezas para asolearlas, colocarlas en el horno y guardarlas. En las familias donde hay jóvenes, ellos dibujan, graban, calan, pintan y diseñan.

En este grupo la mayoría de los artesanos elaboran productos policromados decorativos, 10 hacen grabados y calados, 4 hacen vedes utilitarios, 3 sólo verdes utilitarios, 1 natural decorativo y 1 natural utilitario (ver cuadro 1 ). 
Orientación estratégica y desempeño en un proceso de desarrollo de productos

Alfarería en Santa María Atzompa, Oaxaca, México

Cuadro 1

Descripción de los productos del grupo sujeto de investigación

\begin{tabular}{|c|c|c|}
\hline & Tipo de productos & $\begin{array}{l}\text { No. de } \\
\text { piezas }\end{array}$ \\
\hline 1 & $\begin{array}{l}\text { Decorativa policromada y natural: figuras religiosas, cruces, ángeles, guirnaldas } \\
\text { navideñas, flores, servilleteros. }\end{array}$ & 70 a 100 \\
\hline 2 & $\begin{array}{l}\text { Decorativa policromada: figuras religiosas, cruces, ángeles, guirnaldas, flores, } \\
\text { servilleteros, búhos y fruteros. }\end{array}$ & 50 a 60 \\
\hline 3 & $\begin{array}{l}\text { Decorativa policromados: floreros, tibores, mitades de tibores calados y grabados, } \\
\text { pantallas para lámpara y para veladoras, macetas, cilindros calados } \\
\text { Decorativa verde: macetas } \\
\text { Utilitaria verde: cazuelas }\end{array}$ & 100 a 150 \\
\hline 4 & $\begin{array}{l}\text { Decorativa: macetas naturales y policromada. } \\
\text { Utilitaria verde: cazuelas, jarros, } \\
\text { Utilitaria natural y roja: botellones, ollas para agua }\end{array}$ & 40 a 60 \\
\hline 5 & $\begin{array}{l}\text { Decorativa policromada: cilindros, mitades tibores calados y grabados floreros, } \\
\text { muñecas. }\end{array}$ & 60 a 80 \\
\hline 6 & $\begin{array}{l}\text { Platos policromados grabados, floreros verdes bordados } \\
\text { Utilitaria: cazuelas, jarros, }\end{array}$ & 50 a 70 \\
\hline 7 & $\begin{array}{l}\text { Decorativa policromada: pantallas grabadas y caladas } \\
\text { Utilitaria: cántaros naturales grabados }\end{array}$ & 40 a 60 \\
\hline 8 & $\begin{array}{l}\text { Decorativa policromada: mitades caladas y grabadas, macetas Utilitaria verde: } \\
\text { cazuelas, jarros, platones, apaxtles, salseras, }\end{array}$ & 70 a 100 \\
\hline 9 & $\begin{array}{l}\text { Policromada decorativa, olla bordada y grabada, mariposas policromadas bordadas } \\
\text { y grabadas }\end{array}$ & 40 a 50 \\
\hline 10 & Policromada decorativa: tibores, mitades de tibores calados y utilitaria natural & 40 a 50 \\
\hline 11 & Policromada decorativa: tibores, macetas, jarrones & 25 a 30 \\
\hline 12 & $\begin{array}{l}\text { Utilitaria verde: ollas, jarros, cazuelas, platos, licoreras, copas, servilleteros, salseras, } \\
\text { charolas, y natural }\end{array}$ & 100 a 150 \\
\hline 13 & $\begin{array}{l}\text { Decorativa: floreros policromados grabados, utilitaria verde: tazones verdes } \\
\text { grabados, cazuelas, platos }\end{array}$ & 70 a 80 \\
\hline 14 & Utilitaria verde: cazuela verde grabada, platos verdes extendidos & 50 a 70 \\
\hline 15 & Decorativa: cruces naturales y policromadas, muñecas para nacimiento naturales & 80 a 100 \\
\hline 16 & $\begin{array}{l}\text { Natural decorativa: paloma bordada, mitad natural bordada y grabada, macetas con } \\
\text { forma de elefantes, ranas y utilitaria }\end{array}$ & 30 a 40 \\
\hline 17 & Utilitaria verde: cazuelas & 100 a 150 \\
\hline 18 & $\begin{array}{l}\text { Decorativa policromada: macetas grabadas, cilindros grabados, tibores, pantallas } \\
\text { grabadas y caladas para lámpara y veladora, }\end{array}$ & 50 a 70 \\
\hline 19 & Utilitaria verde: cazuelas, jarros, & 80 a 100 \\
\hline
\end{tabular}

La producción de un lote dura aproximadamente entre 7 y 9 días dependiendo de las condiciones climáticas, pues el calor acelera el secado de las piezas, pero en temporada de sequía también se corre el riesgo de que las piezas se partan y se rompan. 
Cada familia en promedio realiza una o dos quemas al mes, dependiendo de la temporada. La producción es estacional, en octubre elaboran piezas decorativas y utilitarias para venderlas antes de noviembre (día de muertos); otro periodo de producción se realiza en noviembre para las ventas de diciembre, que es cuando hay afluencia turística en Oaxaca; así como en febrero y marzo, antes de la Semana Santa, y en junio para el verano, temporada en la que también hay afluencia turística por las festividades del "Lunes del Cerro" y el periodo vacacional. De manera esporádica, los artesanos — que hacen piezas policromadas— atienden pedidos especiales.

En cada taller hay al menos un horno de acuerdo con las características de las piezas que elaboran; quienes hacen macetas y piezas grandes tienen un horno grande y quienes hacen piezas pequeñas cuentan con un horno pequeño, con una capacidad que depende del tamaño de las piezas y de la habilidad del artesano para acomodar las piezas y utilizar todo el espacio.

Para determinar los problemas de los productos de cerámica se realizó un diagnóstico, revisando someramente 30 piezas en cuanto a pintura, dibujo, esmalte y calado de las piezas. Considerando que las características de un buen producto en artesanía de barro son: resistencia, pulido sin rebaba, colorido, textura homogénea, horneado homogéneo (libre de quemaduras o grietas) y excelente acabado. En las piezas revisadas se observaron problemas de ampollas, aglutinación, pérdida de brillo o lustre de la película de pintura, grietas y descascaramiento, formación de espuma y cráteres, traslape, grietas lodosas, distribución o uniformidad insatisfactorias, cubrimiento deficiente, brillo irregular, corrimiento y arrugas.

En los dibujos se observó poca uniformidad en las líneas con las que graban, líneas que no es necesario pintar cuando existe alto contraste entre el color del dibujo y el color del fondo, poco manejo del espacio (proporción entre el dibujo y el espacio en el que se dibuja); combinación de colores sin contrastes, poca integración de colores (colores que se originan de un solo color como los cafés y naranjas que se originan del rojo), uso de colores muy oscuros o aplicados de manera uniforme que hace que las figuras se vean planas, la pintura demasiado espesa que tiene el mismo efecto y al quemar las piezas se chorrea, el uso de líneas negras para delimitar dibujos.

El proceso de capacitación tuvo como objetivos: rescatar formas y diseños prehispánicos, obtener nuevos diseños de formas y figuras, estimular la creatividad de los artesanos, aprovechar las propiedades de las arcillas en cuanto a textura arenosas y lisas, y aplicar nuevos dibujos a las piezas de barro utilizando pinturas cerámicas. En este proceso se utilizaron procedimientos bien detallados para facilitar la integración de las bases del conocimiento adquirido mediante la capacitación, las habilidades 
Orientación estratégica y desempeño en un proceso de desarrollo de productos

Alfarería en Santa María Atzompa, Oaxaca, México

comunes, los idiomas compartidos y las estructuras cognoscitivas similares que habilitan la comunicación técnica y el aprendizaje en el proceso de producción.

Durante el seguimiento del proceso se levantó una encuesta con el de fin de medir la orientación estratégica y el desempeño; para ello se respondieron las siguientes preguntas de investigación como: ¿cuál es la orientación estratégica de los artesanos? y ¿cómo la orientación estratégica de los artesanos afecta el desempeño de los negocios de artesanía?

Gráfica 1

Orientación estratégica del grupo de artesanos de Santa María Atzompa, Oaxaca

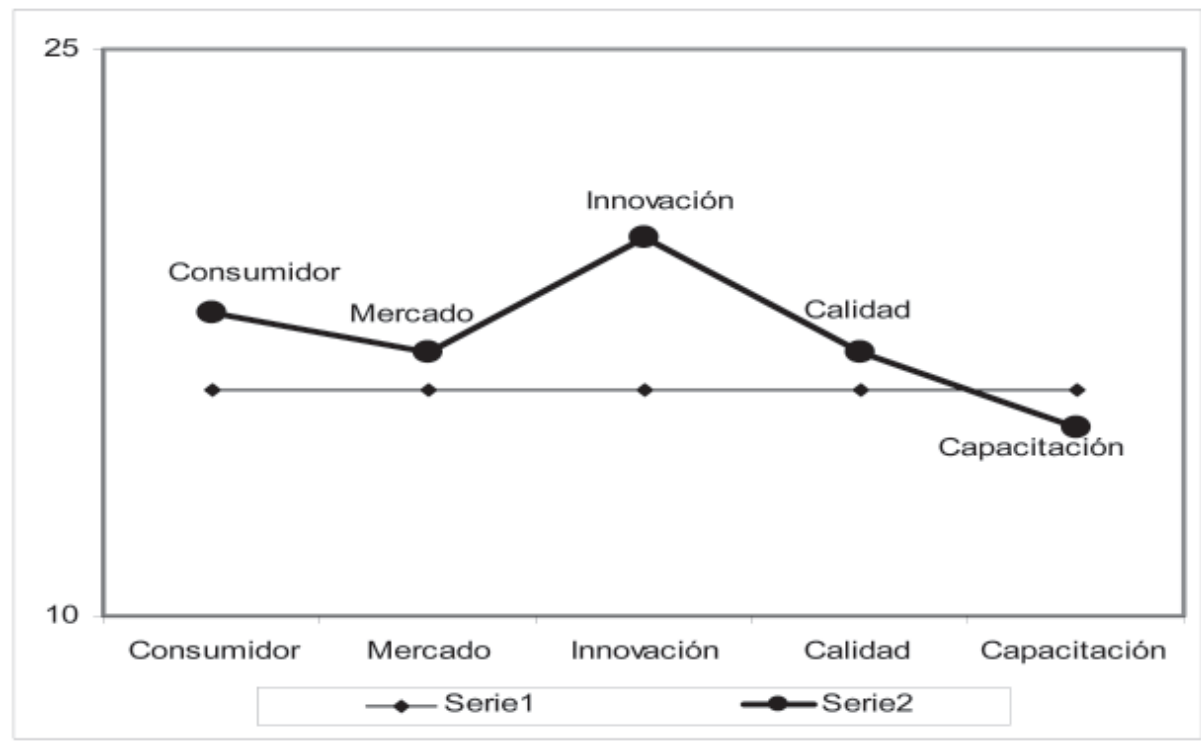


María Luisa Domínguez Hernández, José de la Paz Hernández Girón y Dora Lilia Guzmán Cruz

Cuadro 2

Edad de los artesanos del grupo de innovación

\begin{tabular}{|c|c|c|c|}
\hline Años & Frecuencia & Porcentaje & Acumulado \\
\hline 18 & 1 & 5.3 & 5.3 \\
24 & 1 & 5.3 & 10.5 \\
32 & 1 & 5.3 & 15.8 \\
35 & 1 & 5.3 & 21.1 \\
36 & 1 & 5.3 & 26.3 \\
38 & 1 & 5.3 & 31.6 \\
43 & 1 & 5.3 & 36.8 \\
44 & 1 & 5.3 & 42.1 \\
45 & 1 & 5.3 & 47.4 \\
47 & 1 & 5.3 & 52.6 \\
48 & 1 & 5.3 & 57.9 \\
49 & 2 & 10.5 & 68.4 \\
51 & 1 & 5.3 & 73.7 \\
52 & 1 & 5.3 & 78.9 \\
55 & 1 & 5.3 & 84.2 \\
58 & 1 & 5.3 & 89.5 \\
60 & 1 & 5.3 & 94.7 \\
63 & 1 & 5.3 & 100.0 \\
Total & 19 & 100.0 & \\
\hline
\end{tabular}

Una forma de ver la orientación estratégica de los artesanos es determinando el mapa estratégico, tal como se muestra en la gráfica 1, donde se puede observar que la estrategia de los negocios artesanales está más orientada hacia la innovación y menos hacia la calidad, el mercado y la capacitación.

\section{Muestra}

El trabajo empírico se llevó a cabo en 19 negocios de alfarería con una distribución de edad de los dueños del negocio, como la que se muestra en el cuadro 2, de los cuales el $15 \%$ son hombres y el $85 \%$ son mujeres (ver cuadro 3 ). La escolaridad se muestra en el cuadro 4, donde la mayoría tiene estudios de primaria y sólo dos de bachillerato. 
Orientación estratégica y desempeño en un proceso de desarrollo de productos

Alfarería en Santa María Atzompa, Oaxaca, México

Cuadro 3

Género de los artesanos del grupo de innovación

\begin{tabular}{|c|c|c|c|}
\hline & Frecuencia & Porcentaje & Acumulado \\
\hline Hombre & 3 & 15.8 & 15.8 \\
Mujer & 16 & 84.2 & 100.0 \\
Total & 19 & 100.0 & \\
\hline
\end{tabular}

Cuadro 4

Escolaridad de los artesanos del grupo de innovación

\begin{tabular}{|c|c|c|c|}
\hline & Frecuencia & Porcentaje & Acumulado \\
\hline No sabe leer & 1 & 5.3 & 5.3 \\
Primaria & 13 & 68.4 & 73.7 \\
Secundaria & 3 & 15.8 & 89.5 \\
Bachillerato & 2 & 10.5 & 100.0 \\
Total & 19 & 100.0 & \\
\hline
\end{tabular}

\section{Validación estadística de las escalas}

La conceptualización y la escala de orientación estratégica se diseñaron retomando a González (2003), Morales y Pech (2000) y Ho y Huang (2007); para la validez se utilizó un análisis factorial, determinando la estructura de la variable con cuatro dimensiones (ver cuadro 5): orientación a la innovación, al mercado, a los consumidores y a la capacitación.

Para medir la orientación hacia la calidad de las piezas se utilizaron 42 reactivos que fueron calificados en una escala con valores de cero a diez recodificándola en alta y baja calidad. La evaluación se basó en la observación, se sistematizó de acuerdo con las dimensiones que se muestran en el cuadro 6.

En la figura 1 se esboza el modelo de investigación que refleja el marco teórico, donde se propone el impacto de las variables antecedentes al desempeño.

La varianza explicada en los dos cuadros (5 y 6) nos muestra una alta validez de las escalas utilizadas. 
Cuadro 5

Análisis factorial de orientación estratégica

\begin{tabular}{|c|c|c|c|c|c|}
\hline & 1 & 2 & 3 & 4 & Comunalidad \\
\hline \multicolumn{6}{|l|}{ 1. Orientación estratégica a la innovación } \\
\hline Productos con acabado más fino & .821 & .335 & .327 & .032 & .895 \\
\hline Mejoras en colores & .809 & .335 & .276 & .095 & .852 \\
\hline Mejoras en formas & .755 & .337 & .453 & .071 & .893 \\
\hline Mejoras en dibujos & .898 & .165 & .356 & .097 & .969 \\
\hline Mejoras en barnices & .727 & .149 & .420 & .238 & .784 \\
\hline Productos más atractivos & .808 & .471 & .206 & .195 & .956 \\
\hline Productos más vistosos & .840 & .419 & .182 & .196 & .953 \\
\hline Productos más novedosos & .759 & .401 & .271 & .250 & .873 \\
\hline Información sobre el mercado & .700 & .347 & -.193 & .147 & .670 \\
\hline Capacitación para mejorar sus productos & .700 & .424 & .347 & .318 & .891 \\
\hline $\begin{array}{l}\text { Cambios en el volumen de } \\
\text { producción del año anterior }\end{array}$ & .799 & -.131 & .409 & .078 & .828 \\
\hline Disponibilidad para producir más y mejor & .747 & .087 & .517 & .297 & .921 \\
\hline Cursos de capacitación sobre producción en el año & .889 & .168 & .162 & .255 & .910 \\
\hline \multicolumn{6}{|l|}{ 2. Orientación estratégica al mercado } \\
\hline Seguimiento a los clientes & .098 & .897 & .297 & .056 & .905 \\
\hline Estudios de mercado realizados & .102 & .852 & .359 & .087 & .873 \\
\hline Buscar nuevos clientes y nuevos mercados & .178 & .758 & .196 & .396 & .801 \\
\hline Información sobre diseños de la competencia & .368 & .838 & .043 & .061 & .843 \\
\hline $\begin{array}{l}\text { Interés por el comportamiento de los productos } \\
\text { en el mercado }\end{array}$ & .568 & .671 & .285 & .203 & .896 \\
\hline $\begin{array}{l}\text { Comunicación con otros artesanos } \\
\text { sobre comportamiento de los productos }\end{array}$ & .389 & 689 & .494 & .070 & .875 \\
\hline $\begin{array}{l}\text { Comunicación con otros artesanos sobre los productos } \\
\text { por vender }\end{array}$ & .463 & .613 & .330 & .075 & .704 \\
\hline \multicolumn{6}{|l|}{ 3. Orientación estratégica hacia los consumidores } \\
\hline $\begin{array}{l}\text { Información sobre las preferencias del consumidor de } \\
\text { sus productos }\end{array}$ & .342 & .474 & .758 & -.011 & .917 \\
\hline $\begin{array}{l}\text { Información sobre los gustos del consumidor de sus } \\
\text { productos }\end{array}$ & .294 & .409 & .785 & .084 & .877 \\
\hline $\begin{array}{l}\text { Información directa sobre gustos y preferencias del } \\
\text { consumidor }\end{array}$ & .357 & .394 & .798 & .138 & .938 \\
\hline $\begin{array}{l}\text { Acciones como respuestas a las sugerencia de los } \\
\text { consumidores }\end{array}$ & .458 & .504 & .636 & .187 & .904 \\
\hline Manejo de precios como respuesta a la competencia & .270 & .190 & .625 & 614 & .877 \\
\hline \multicolumn{6}{|l|}{ 4. Orientación estratégica a la capacitación } \\
\hline Cursos de capacitación recibidos & .410 & .212 & .059 & .845 & .930 \\
\hline Varianza total explicada & 37.6 & 24.1 & 18.3 & 7.21 & 87.44 \\
\hline
\end{tabular}

Método de extracción: Análisis de componentes principales

Método de rotación: Normalización Varimax con Kaiser

La rotación ha convergido en 7 iteraciones. 
Orientación estratégica y desempeño en un proceso de desarrollo de productos

Alfarería en Santa María Atzompa, Oaxaca, México

\section{Resultados}

La evaluación del impacto de calidad sobre el desempeño se realizó con una prueba $t$ Student con muestras independientes (ver cuadro 7). Sólo la calidad en terminado, en pintura y dibujo, presentaron una diferencia significativa con respecto las dimensiones de desempeño. La calidad en pintura se relacionó únicamente con las utilidades, mientras que la calidad en dibujo presenta diferencia significativa sólo con ventas. La dimensión más importante es la de calidad en acabado de las piezas de cerámica porque muestra diferencia significativa muy alta y con las dos dimensiones de desempeño, lo que explica la capacidad de orientación estratégica que se despertó en los artesanos durante el periodo de trabajo.

Cuadro 6

Análisis factorial de orientación estratégica hacia la calidad

\begin{tabular}{|c|c|c|c|c|c|c|}
\hline & 1 & 2 & 3 & 4 & 5 & Comunalidad \\
\hline \multicolumn{7}{|l|}{ 1. Calidad en el terminado de la pieza } \\
\hline Delineado del contorno del grabado & .919 & .136 & -.039 & .220 & .063 & .917 \\
\hline $\begin{array}{l}\text { Uniformidad en la profundidad } \\
\text { del grabado }\end{array}$ & .869 & .279 & -.097 & .206 & .021 & .884 \\
\hline Remarcado, líneas sobrepuestas & .909 & .016 & .178 & .321 & .023 & .962 \\
\hline $\begin{array}{l}\text { Uniformidad en el grosor de la } \\
\text { línea grabada }\end{array}$ & .933 & .070 & .017 & .193 & .030 & .915 \\
\hline Grosor & .775 & .300 & .032 & .091 & .403 & .862 \\
\hline Cocción & .774 & .375 & .246 & .079 & .390 & .958 \\
\hline Filtración & .697 & .491 & .245 & -.106 & .343 & .914 \\
\hline Sonoridad & .748 & .422 & .237 & .100 & .241 & .862 \\
\hline Acabado & .775 & .279 & .389 & .078 & .330 & .944 \\
\hline Grumos en la pieza & .791 & .386 & .235 & .046 & .372 & .971 \\
\hline Fisuras en la pieza & .817 & .419 & .262 & .004 & .212 & .958 \\
\hline Armonía visual de toda la pieza & .770 & .320 & .304 & .090 & .383 & .944 \\
\hline \multicolumn{7}{|l|}{ 2. Calidad en el esmalte } \\
\hline Uniformidad del esmalte & .267 & .837 & .324 & .046 & .118 & .893 \\
\hline Distribución del esmalte & .244 & .888 & .216 & -.044 & .189 & .932 \\
\hline Craquelado & .245 & .878 & .232 & .202 & .186 & .960 \\
\hline Manchado & .248 & .839 & .324 & .180 & .038 & .905 \\
\hline Escarapelado & .304 & .869 & .342 & .157 & .072 & .994 \\
\hline Partes con esmalte crudo & .252 & .820 & .281 & .106 & .248 & .887 \\
\hline Partes quemadas & .252 & .794 & .413 & .241 & .190 & .958 \\
\hline Partes ahumadas & .258 & .783 & .406 & .192 & .196 & .920 \\
\hline Brillo & .260 & .780 & .412 & .248 & .081 & .914 \\
\hline Densidad & .241 & .869 & .276 & .032 & .232 & .944 \\
\hline
\end{tabular}




\begin{tabular}{|c|c|c|c|c|c|c|}
\hline \multicolumn{7}{|l|}{ 3. Calidad en pintura: } \\
\hline Corrimiento & .149 & .384 & .874 & .165 & .071 & .966 \\
\hline Traslape y problemas de marco & .166 & .373 & .849 & .265 & .073 & .963 \\
\hline Cubrimiento & .169 & .384 & .829 & .337 & .059 & .981 \\
\hline Relación color objeto (Acertividad) & .154 & .363 & .856 & .293 & .091 & .982 \\
\hline Uniformidad en los colores & .158 & .382 & .832 & .306 & .069 & .962 \\
\hline Contraste & .147 & .374 & .841 & .334 & .085 & .986 \\
\hline Distribución & .080 & .306 & .794 & .284 & .367 & .862 \\
\hline Uniformidad del engobe & .080 & .306 & .794 & .284 & .367 & .946 \\
\hline \multicolumn{7}{|l|}{ 4. Calidad en el calado } \\
\hline Traslape de la línea con el calado & .164 & .123 & .277 & .928 & .081 & .987 \\
\hline Despostillado en la orilla del calado & .164 & .123 & .277 & .928 & .081 & .987 \\
\hline Simetría en los cortes & .164 & .123 & .277 & .928 & .081 & .987 \\
\hline Rebabas en el calado & .147 & .143 & .267 & .883 & .022 & .894 \\
\hline Precisión de la línea & .168 & .108 & .271 & .918 & .076 & .961 \\
\hline \multicolumn{7}{|l|}{ 5. Calidad en el dibujo } \\
\hline Nitidez del dibujo & .497 & .397 & .281 & .178 & .632 & .915 \\
\hline Precisión del trazo & .520 & .421 & .143 & .045 & .693 & .951 \\
\hline Nitidez y claridad en la figura & .594 & .253 & .175 & .133 & .703 & .959 \\
\hline Proporción dibujo-pieza & .594 & .253 & .175 & .133 & .703 & .959 \\
\hline $\begin{array}{l}\text { Perspectiva (Distancia, } \\
\text { profundidad y volumen) }\end{array}$ & .582 & .180 & .243 & .103 & .719 & .957 \\
\hline Varianza total explicada & 26.3 & 24.6 & 19.5 & 13.7 & 9.84 & 94.209 \\
\hline
\end{tabular}

Método de extracción: Análisis de componentes principales

Método de rotación: Normalización Varimax con Kaiser

La rotación ha convergido en 9 iteraciones

En el cuadro 8 puede observarse el efecto que tuvo la orientación en innovación sobre las ventas y las utilidades. La diferencia entre los dos grupos es significativa; es decir, los que realizaron mayor innovación tuvieron un promedio de ventas más alto durante el año de trabajo, $(\$ 34,107.14$ contra $\$ 20,000.00)$, además reportaron más utilidades $(\$ 5,225.15$ contra $\$ 2,408.31)$. También puede apreciarse cómo el efecto de los que más innovaron es mayor en las utilidades que en las ventas, pues tienen una significancia mayor $(0.002$, contra 0.000$)$. 
Orientación estratégica y desempeño en un proceso de desarrollo de productos

Alfarería en Santa María Atzompa, Oaxaca, México

Cuadro 7

Prueba t de muestras independientes con calidad y desempeño

\begin{tabular}{|c|c|c|c|c|c|c|c|c|}
\hline & $\begin{array}{c}\text { Calidad } \\
\text { Total }\end{array}$ & $\mathbf{N}$ & Media & Desviación típica & Error típico & t & gl & Significancia \\
\hline \multirow[t]{2}{*}{ Ventas } & 1 & 13 & 27115.3846 & 9721.44075 & 2696.24255 & -2.058 & 17 & .055 \\
\hline & 2 & 6 & 37500.0000 & 11344.60224 & 4631.41447 & -1.938 & 8.554 & \\
\hline \multirow[t]{3}{*}{ Utilidades } & 1 & 13 & 11615.3846 & 5560.85218 & 1542.30290 & -2.525 & 17 & .022 \\
\hline & 2 & 6 & 18166.6667 & 4445.97196 & 1815.06045 & -2.751 & 12.181 & \\
\hline & $\begin{array}{c}\text { Calidad } \\
\text { en Pintura }\end{array}$ & & & & & & & \\
\hline \multirow[t]{2}{*}{ Ventas } & 1 & 9 & 26388.8889 & 7105.35792 & 2368.45264 & -1.548 & 17 & .140 \\
\hline & 2 & 10 & 34000.0000 & 13089.43594 & 4139.24309 & -1.596 & 14. & \\
\hline \multirow[t]{2}{*}{ Utilidades } & 1 & 9 & 10888.8889 & 4371.62568 & 1457.20856 & -2.105 & 17 & .050 \\
\hline & 2 & 10 & 16200.0000 & 6321.04070 & 1998.88858 & -2.147 & 16 & \\
\hline & $\begin{array}{c}\text { Calidad } \\
\text { en dibujo }\end{array}$ & & & & & & & \\
\hline \multirow[t]{2}{*}{ Ventas } & 1 & 8 & 24437.5000 & 7724.90176 & 2731.16521 & -2.195 & 17 & .042 \\
\hline & 2 & 11 & 34727.2727 & 11455.05049 & 3453.82768 & -2.337 & 16 & \\
\hline \multirow[t]{3}{*}{ Utilidades } & 1 & 8 & 11750.0000 & 5496.75229 & 1943.39541 & -1.216 & 17 & .241 \\
\hline & 2 & 11 & 15090.9091 & 6187.96486 & 1865.74160 & -1.240 & 16 & \\
\hline & $\begin{array}{c}\text { Calidad } \\
\text { en acabado }\end{array}$ & & & & & & & \\
\hline \multirow[t]{2}{*}{ Ventas } & 1 & 7 & 23500.0000 & 23500.0000 & 7354.13716 & -2.300 & 17 & .034 \\
\hline & 2 & 12 & 34416.6667 & 34416.6667 & 11155.58020 & -2.566 & 16.602 & \\
\hline \multirow[t]{2}{*}{ Utilidades } & 1 & 7 & 9714.2857 & 9714.2857 & 4855.04156 & -2.510 & 17 & .022 \\
\hline & 2 & 12 & 16000.0000 & 16000.0000 & 5477.22558 & -2.595 & 14.005 & . \\
\hline
\end{tabular}

Cuadro 8

Prueba t orientación a la innovación

Prueba de muestras independientes

\begin{tabular}{|ll|r|r|r|r|r|r|r|}
\hline & innovación & \multicolumn{1}{|c|}{ N } & Media & $\begin{array}{l}\text { Desviación } \\
\text { típica }\end{array}$ & $\begin{array}{l}\text { Error típico } \\
\text { de la media }\end{array}$ & \multicolumn{1}{c|}{ t } & \multicolumn{1}{c|}{ gl } & Significancia \\
\hline Ventas & 1 & 5 & $20,000.00$ & $5,431.39$ & 2428.99156 & -2.895 & 17 & .010 \\
& 2 & 14 & $34,107.14$ & $10,262.28$ & 2742.71191 & -3.851 & 13.800 & \\
Utilidades & 1 & 5 & $7,400.00$ & $2,408.31$ & 1077.03296 & -3.471 & 17 &. $\mathbf{0 0 3}$ \\
& 2. & 14 & $15,928.57$ & $5,225.15$ & 1396.48021 & -4.836 & 15.380 & \\
\hline
\end{tabular}

Cuadro 9

Prueba t orientación a los consumidores

Prueba de muestras independientes

\begin{tabular}{|l|c|r|l|r|r|r|r|r|}
\hline & $\begin{array}{c}\text { Orientación } \\
\text { a los } \\
\text { consumidores }\end{array}$ & N & Media & $\begin{array}{l}\text { Desviación } \\
\text { típica }\end{array}$ & Error típico & \multicolumn{1}{c|}{$\mathbf{t}$} & \multicolumn{1}{c|}{ gl } & Significancia \\
\hline Ventas & 1 & 8 & $23,625.00$ & $80,52.28$ & 2846.91249 & -2.605 & 17 & .019 \\
& 2 & 11 & $35,318.18$ & $106,44.88$ & 3209.55443 & -2.726 & 16.943 & \\
Utilidades & 1 & 8 & $11,250.00$ & $56,25.19$ & 1988.80797 & -1.571 & 17 & .135 \\
& 2 & 11 & $15,454.54$ & $58,54.29$ & 1765.13526 & -1.581 & 15.597 & \\
\hline
\end{tabular}


La orientación al mercado sólo tiene efecto sobre las utilidades reportadas por el grupo de cerámica (ver cuadro 10). La diferencia de medias en ventas es casi significativa (.07), mientras que el promedio de las utilidades de los dos grupos presenta una diferencia significativa a nivel de .02 , siendo mayor el promedio de utilidades de los que tienen mayor orientación hacia el mercado (\$15,692.30 contra \$9,333.33). Esto muestra que la orientación al mercado es una estrategia muy importante para generar más utilidades en el grupo de cerámica de Santa María Atzompa.

\section{Cuadro 10}

Prueba t orientación al mercado Prueba de muestras independientes

\begin{tabular}{|l|c|r|l|r|r|r|r|r|}
\hline & $\begin{array}{l}\text { Orientación } \\
\text { al mercado }\end{array}$ & \multicolumn{1}{|c|}{$\mathbf{N}$} & Media & $\begin{array}{c}\text { Desviación } \\
\text { típica }\end{array}$ & \multicolumn{1}{c|}{ Error típico } & \multicolumn{1}{c|}{$\mathbf{t}$} & \multicolumn{1}{c|}{ gl } & Significancia \\
\hline Ventas & 1 & 6 & $23,833.33$ & $9,683.31$ & 3953.19842 & -1.866 & 17 & .079 \\
& 2 & 13 & $33,423.07$ & $10,700.16$ & 2967.69257 & -1.940 & 10.795 & \\
Utilidades & 1 & 6 & $9,333.33$ & $4,633.21$ & 1891.50146 & -2.424 & 17 & $\mathbf{. 0 2 7}$ \\
& 2 & 13 & $15,692.30$ & $5,573.51$ & 1545.81576 & -2.603 & 11.729 & \\
\hline
\end{tabular}

En el cuadro 11 se muestra cómo la orientación a la capacitación no tiene efecto sobre los resultados del grupo de cerámica, pues ninguna de las diferencias de medias es significativa. Esto denota que a pesar de que son muchos los que tomaron más cursos de capacitación (15 contra 4) no presentan diferencia significativa con el promedio de ventas y utilidades de los que tomaron menos cursos de capacitación, lo que muestra que los cursos sobre dibujo y pintura presentan poco efecto para el desarrollo del producto de cerámica; también se explica por qué los cursos que reportan los artesanos no son sólo los que este grupo impartió, sino que reportan todos los cursos recibidos en su vida. Lo que si queda claro es que no existe impacto de la capacitación sobre las ventas ni las utilidades del grupo de cerámica de Santa María Atzompa.

Cuadro 11

Prueba t orientación a la capacitación Prueba de muestras independientes

\begin{tabular}{|l|c|r|l|r|r|r|r|r|}
\hline & $\begin{array}{c}\text { Orientación a } \\
\text { la capacitación }\end{array}$ & $\mathbf{N}$ & Media & \multicolumn{1}{c|}{$\begin{array}{l}\text { Desviación } \\
\text { tíca }\end{array}$} & Error típico & t & \multicolumn{1}{c|}{$\mathbf{g l}$} & Significancia \\
\hline Ventas & 1 & 4 & $26,250.00$ & 16700.79 & 8350.39919 & -.833 & 17 & .416 \\
& 2 & 15 & $31,500.00$ & 9623.26 & 2484.71518 & -.603 & 3.549 & \\
Utilidades & 1 & 4 & $11,750.00$ & 7847.50 & 3923.75246 & -.717 & 17 & .483 \\
& 2 & 15 & $14,200.00$ & 5621.38 & 1451.43607 & -.586 & 3.862 & \\
\hline
\end{tabular}


Orientación estratégica y desempeño en un proceso de desarrollo de productos

Alfarería en Santa María Atzompa, Oaxaca, México

\section{Conclusiones}

En la figura 2 se observa cómo la orientación a la capacitación queda fuera del modelo, la orientación a la calidad y hacia la innovación son las variables que más efecto tiene sobre el desempeño, y la orientación al mercado y a los consumidores sólo afectan las utilidades de los negocios de alfarería en Santa María Atzompa.

Figura 2

Diagrama resultado del análisis estadístico

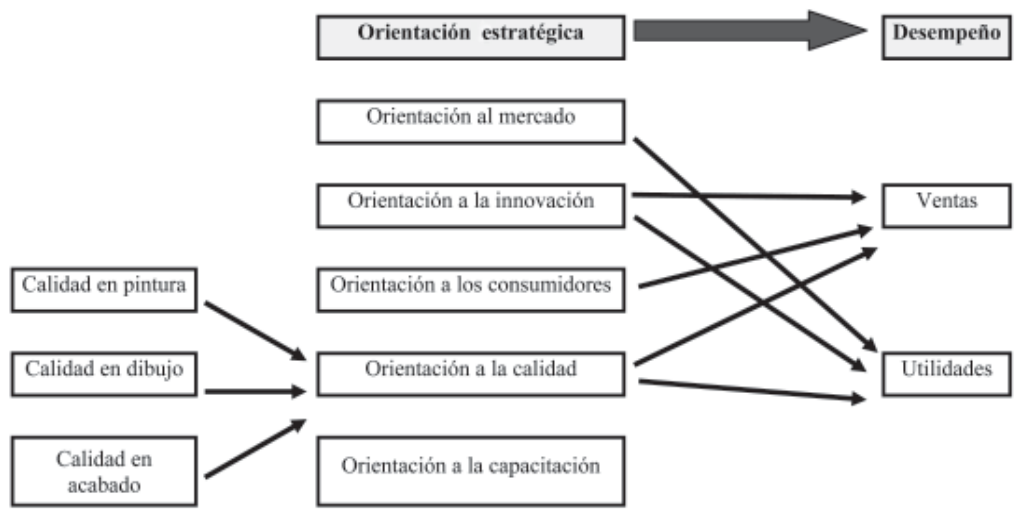

Los artesanos le dan mucha importancia al acabado final de las piezas, manifestando su preocupación por la calidad en cuanto al grabado (delineado del contorno, uniformidad en la profundidad, remarcado, líneas sobrepuestas, uniformidad en el grosor de la línea grabada), cocción, filtración, sonoridad, acabado, grumos en la pieza, fisuras en la pieza, armonía visual de toda la pieza; además del traslape de la línea con el calado, despostillado en la orilla del calado, simetría en los cortes, rebabas en el calado y precisión de la línea.

Por otro lado, los problemas que los artesanos tenían con el barniz parece que los superaron al menos en cuanto a uniformidad del esmalte, distribución del esmalte, craquelado, manchado, escarapelado, partes con esmalte crudo, partes quemadas, partes ahumadas, brillo y densidad.

La orientación a la innovación y a la calidad son las estrategias más importante para los artesanos, porque afectan tanto a las ventas como a las utilidades, mientras que la orientación a los consumidores sólo tiene efecto sobre las ventas y la orientación al mercado sólo afecta las utilidades. En consecuencia, se recomienda que en la capacitación se haga énfasis en la orientación al mercado con estrategias para dar mejor seguimiento a los clientes, realizar estudios de mercado, buscando nuevos mercados, información sobre la competencia, despertando más interés por el comportamiento 
de los productos en el mercado y por la comunicación con otros artesanos sobre comportamiento de los productos. También debe mejorarse la orientación a los consumidores, obteniendo información sobre las preferencias y gustos del consumidor, diseñando estrategias para dar respuestas a las sugerencias de los consumidores y a la competencia.

La explicación del por qué la orientación estratégica hacia la capacitación no tuvo un efecto significativo sobre el desempeño de los negocios de cerámica se puede centrar, posiblemente, en que se consideró esa orientación parte de la planificación proactiva, que como dice Burack (1990) conduce a la renovación crecimiento y desarrollo de la fuerza de trabajo. Asimismo, se midió con reactivos que permitieran determinar si a los productores les interesaba encontrar nuevas forma de organización, nuevas formas de distribución de los productos, búsqueda de nuevos mercados y con que frecuencia realizaban cambios en la organización del trabajo. Dichos reactivos no pasaron la prueba de validez y sólo quedaron los cursos tomados.

\section{Bibliografía}

Aragón, A. y G. SÁnchez (2005), "Strategic Orientation, Management Characteristics and Performance: A Study of Spanish SMES, Journal of Small Business Management, 43(3), 287-308.

BARNEY, J. (1991), "Firm resources and sustained competitive advantage", Journal of Management, vol. 17 (1), 99-120.

Brouthers, K., M- Gelderman y P. Arens (2007), "The Influence of ownership on performance: stakeholder and straegIc contingency perspectives", Schmalenbach Business Review, 59, 225-242.

Burack, Elmer H. (1990), Planificación y aplicaciones creativas de recursos humanos. Una orientación estratégica, Ediciones Díaz de Santos, Madrid, 485-509.

Eddleston, K., F. Kellermans y R. Sarathy (2008), "Resource Configuration in Family Firms: Linking Resources, Strategic Planning and Technological Opportunities to Performance", Journal of Management Studies, 45(1), 26-50.

GonZÁLEZ, Eduardo (2003), "Los factores de competitividad: el efecto grupo", Revista CEPADE, 29(3), 28-41.

Hernández, J., M. Domínguez y M. Caballero (2007), "Factores de innovación en negocios de artesanía de México", Gestión y Política Pública, XVI(2), 353-379.

y J. JiMÉnEZ (2007), "Strategy and factors for success: The mexican handicraft sector", Performance Inprovement Journal, 46(8),16-26. 
Orientación estratégica y desempeño en un proceso de desarrollo de productos

Alfarería en Santa María Atzompa, Oaxaca, México

Ho \& HuANG, Ch. (2007), "Market Orientation Strategies and Business Performance: Evidence from Taiwan's Life Insurance Industry", Journal of American Academy of Business, Cambridge, 11(1), 297-302.

Hunt S.D, y R. M. Morgan (1995), "The comparative advantage theory of competition", Journal of Marketing, 59, 1-15.

KoHLI A.K. y B. J. JAWORSKI (1990), "Market orientation: the construct, research propositions and managerial implications", Journal of Marketing, 54, 1-18.

LinARes, C., y A. Acevedo (2006), "El concepto de innovación tecnológica en servicios de telecomunicaciones", Revista de Telecomunicaciones, 105, 49- 69.

Marlin, D., D. Ketchen y B. Lamont (2007), "Equifinality and the Strategic GroupsPerformance Relationship”, Journal of Managerial Issues, 19 (2), 208-233.

Mavondo, Felix T. (1999), "Environment and strategy as antecedents for marketing effectiveness and organizational performance", Journal of Strategic Marketing, $7,237-250$.

MezA, Mónica del Carmen M. (2005), "Modelos de pedagogía empresarial”, Educación y educadores, 8(1), 78-89.

MintzBerg, H. (1987), “Crafting strategy”, Harvard Business Review, julio-agosto, 6-75.

Morales, M., y J. Pech (2000), "Competitividad y estrategia: el enfoque, de las competencias esenciales y el enfoque basado en los recursos", Contaduría y Administración, 197, División de Investigación, FCA, UNAM, 43-63.

Morgan, R. y C. Strong (1998), Market orientation and dimensions of strategic orientation, European Journal of Marketing, 32(1112), 1051-1073.

PLESHKO, L. y N. SOULDEN (2003), "The profit effects of product-market growth strategy: A financial services example", Journal of Financial Services Marketing, 7(3), $258-266$.

Rodríguez, A. (2005), "Un modelo integral para evaluar el impacto de la transferencia de conocimiento interorganizacional en el desempeño de la firma", Estudios Gerenciales, 095, 37-50.

Salavou H. G. Baltas y S. Lioukas (2004), "Organizational innovation in SMEs. The importance of strategic orientation and competitive structure", European journal of marketing, 38(9-10), 1091-1112.

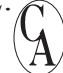

